\title{
Construction of a comprehensive endovascular test bed for research and device development in mechanical thrombectomy in stroke
}

\author{
Adithya S. Reddy, BSE,' Yang Liu, PhD, ${ }^{2}$ Joshua Cockrum, BSE,' Daniel Gebrezgiabhier, BSE,1 \\ Evan Davis, BSE, ${ }^{2}$ Yihao Zheng, PhD, ${ }^{3}$ Aditya S. Pandey, MD, ${ }^{1}$ Albert J. Shih, PhD, ${ }^{2}$ and \\ Luis E. Savastano, MD, PhD ${ }^{1,4}$
}

Departments of ${ }^{1}$ Neurosurgery and ${ }^{2}$ Mechanical Engineering, University of Michigan, Ann Arbor, Michigan; ${ }^{3}$ Department of Mechanical Engineering, Worcester Polytechnic Institute, Worcester, Massachusetts; and ${ }^{4}$ Department of Neurosurgery, Mayo Clinic, Rochester, Minnesota

OBJECTIVE The development of new endovascular technologies and techniques for mechanical thrombectomy in stroke has greatly relied on benchtop simulators. This paper presents an affordable, versatile, and realistic benchtop simulation model for stroke.

METHODS A test bed for embolic occlusion of cerebrovascular arteries and mechanical thrombectomy was developed with 3D-printed and commercially available cerebrovascular phantoms, a customized hydraulic system to generate physiological flow rate and pressure, and 2 types of embolus analogs (elastic and fragment-prone) capable of causing embolic occlusions under physiological flow.

RESULTS The test bed was highly versatile and allowed realistic, radiation-free mechanical thrombectomy for stroke due to large-vessel occlusion with rapid exchange of geometries and phantom types. Of the transparent cerebrovascular phantoms tested, the 3D-printed phantom was the easiest to manufacture, the glass model offered the best visibility of the interaction between embolus and thrombectomy device, and the flexible model most accurately mimicked the endovascular system during device navigation. None of the phantoms modeled branches smaller than $1 \mathrm{~mm}$ or perforating arteries, and none underwent realistic deformation or luminal collapse from device manipulation or vacuum. The hydraulic system created physiological flow rate and pressure leading to iatrogenic embolization during thrombectomy in all phantoms. Embolus analogs with known fabrication technique, structure, and tensile strength were introduced and consistently occluded the middle cerebral artery bifurcation under physiological flow, and their interaction with the device was accurately visualized.

CONCLUSIONS The test bed presented in this study is a low-cost, comprehensive, realistic, and versatile platform that enabled high-quality analysis of embolus-device interaction in multiple cerebrovascular phantoms and embolus analogs. https://thejns.org/doi/abs/10.3171/2020.1.JNS192732

KEYWORDS cerebrovascular simulator; embolus; large-vessel occlusion; cerebral artery; stroke; thrombectomy; 3D printing; vascular disorders; interventional neurosurgery

$\mathrm{T}$ HE development of new endovascular technologies and techniques for mechanical thrombectomy in stroke have largely relied on the use of benchtop simulators. ${ }^{1,2}$ Notwithstanding the relevance for continued widespread progress, most of the published data on techniques for the manufacture of cerebral artery phantoms do not provide details of the assembly and operation of the entire pressurized circuit and how to manufacture realistic embolus analogs (EAs) to replicate the phenomenon seen in patients with stroke. ${ }^{3}$ Moreover, published manufacturing techniques for EAs use animal blood and therefore are not readily available, cause animal discomfort, and/or are expensive. In addition, the published literature does not provide a comparative analysis of the advantages and disadvantages of the different types of cerebrovascular phantoms, nor does it address the ideal use of a particular simu-

ABBREVIATIONS CAD = computer-aided design; CAPTIVE = continuous aspiration prior to intracranial vascular embolectomy; EA = embolus analog; ICA = internal carotid artery; ID = inner diameter; LVO = large-vessel occlusion; $M C A=$ middle cerebral artery; $P V C=$ polyvinyl chloride; RBC = red blood cell; $S T L=$ stereolithography; $\varepsilon=$ strain; $\varepsilon_{u t}=$ ultimate tensile strain; $\sigma=$ stress; $\sigma_{u t}=$ ultimate tensile stress.

SUBMITTED October 9, 2019. ACCEPTED January 28, 2020.

INCLUDE WHEN CITING Published online April 3, 2020; DOI: 10.3171/2020.1.JNS192732. 
lator to test a specific feature of the thrombectomy device. This is critical because results obtained in a particular phantom may over- or underrepresent the occurrence of a certain phenomenon, leading to incorrect findings that will drive suboptimal device development. Moreover, the cerebrovascular phantoms in use are not generally transparent and rely on the use of radiographs, exposing the operators to radiation, driving up costs by requiring an angiography unit, and potentially missing critical information during embolus removal.

To fill this gap, this paper presents the following information: 1) the process of manufacturing highly accurate experimental setups that mimic with high fidelity the angioarchitecture of brain arteries based on 3D printing; 2) commercially available vascular phantoms of different materials for radiation-free visualization, with the advantages and disadvantages of each type; 3 ) the fabrication of a hydraulic system with tailored flow resistance to generate physiological flow within any of these cerebral phantoms and to mimic cerebrovascular hemodynamic conditions; 4) the technique to generate EAs from banked human blood that can be introduced into the arterial system to cause an embolic occlusion that withstands physiological flow in these phantoms; and 5) tensile characterization of the EAs. It is our expectation that this paper will be of value to researchers, interventionalists, and companies interested in establishing an affordable, versatile, and realistic benchtop cerebrovascular and stroke simulator and in understanding the pros and cons of available options for arterial phantoms and EAs.

\section{Methods}

A test bed for embolic occlusion of cerebrovascular arteries and mechanical thrombectomy was developed with 1) cerebrovascular phantom, 2) hydraulic system, and 3) EAs.

\section{Cerebrovascular Phantoms}

The cerebrovascular phantoms in this study have anatomically accurate arterial lumen geometries and are built with transparent or semitransparent material to enable the visualization of embolus-device interaction without the use of ionizing radiation.

The reconstruction of the arterial lumen includes identification, isolation, reconstruction, and postprocessing of CTA scans of stroke patients. A 5-step process was used (Fig. 1A). In step 1, de-identified patient CTA images were saved in DICOM format and then imported into visualization and medical image computing software-3D Slicer (version 4.8.1; http://www.slicer.org) - to render the cerebral vascular 3D geometry. ${ }^{4}$ In 3D Slicer, the "CTCoronary-Arteries-3" preset and the Visualization Toolkit Graphics Processing Unit (VTK GPU) ray-casting setting were used to render the volume. In step 2, the internal carotid artery (ICA) and the main intracranial branches were isolated from the surrounding tissue. In 3D Slicer, "Threshold effects" was used to highlight the arterial segment of interest and remove all nonvascular tissue. In step 3 , the arterial segment of interest was exported as a stereolithography (STL) model. This model used triangular planes to represent the surface and was rough with imperfections. The STL model was then imported into SolidWorks (version 2018; Dassault Systèmes) for postprocessing to remove those imperfections. In step 4, the arterial path was delineated. Center points along the lengths of the arteries were added and then connected smoothly using a spline. In step 5, the cross-sections along the arterial path were created. For each cross-section, the boundary of the arterial lumen was delineated with approximately 10 points and then connected smoothly, using the spline and the "Loft" command in SolidWorks, to generate the 3D computer-aided design (CAD) model.

To ensure that reconstruction and postprocessing did not change the size of the arterial lumen, the dimensions of the arterial lumen at the ICA and its main branches were directly measured from the CTA image and compared to those in the final CAD model. With this reconstructed arterial lumen geometry, 3 types of phantoms were fabricated.

\section{D-Printed Phantom}

A block with internal channels matching the reconstructed arterial lumen geometry was 3D-printed using an STL 3D printer (Form 2; Formlabs) with $25-\mu$ m layer height (Fig. 1B). With this machine it is also possible to print the freestanding vascular geometry. The block with internal channels (i.e., arterial lumen) was selected over the freestanding vascular geometry due to less refraction and better visualization. Inlet and outlet ports were added to the CAD model and printed. The block was printed using Clear Resin (Formlabs) to create an optically transparent vascular phantom. The surfaces of the block were then smoothed with sandpaper, and Colormaster Acrylic Crystal Clear (Krylon) was used to apply a clear gloss coat to increase transparency. This 3D-printing technology enables fast (approximately 2 days) and relatively cheap (approximately US\$3500 printer cost and < US\$30 material cost for one phantom) fabrication of cerebrovascular phantoms.

\section{Glass Phantom}

A glass phantom matching the reconstructed arterial lumen geometry was fabricated by Farlow's Scientific Glassblowing, Inc., as shown in Fig. 1C. The phantom is a freestanding glass wall made of borosilicate that was shaped by hand. The ICA outer diameter was $8 \mathrm{~mm}$ and the wall thickness was $1 \mathrm{~mm}$. A glass tube with an outer diameter of $3 \mathrm{~mm}$ and a wall thickness of $0.7 \mathrm{~mm}$ was attached to the distal end of the phantom and tapered to replicate the 3D geometry of the middle cerebral artery (MCA). The cost of a custom-made phantom of the cervical ICA, the ICA terminus, and the MCA candelabra, including $\mathrm{M}_{3}$ branches, is approximately US $\$ 3000$.

\section{Silicone Phantom}

A flexible phantom with the arterial wall made of silicone (Fig. 1D) was fabricated by Vascular Simulations. The silicone used has a durometer, wall thickness, and elastic modulus within physiological range, according to the manufacturer. To assemble this phantom, an acrylic box was constructed with a laser-cut quarter-inch acrylic 

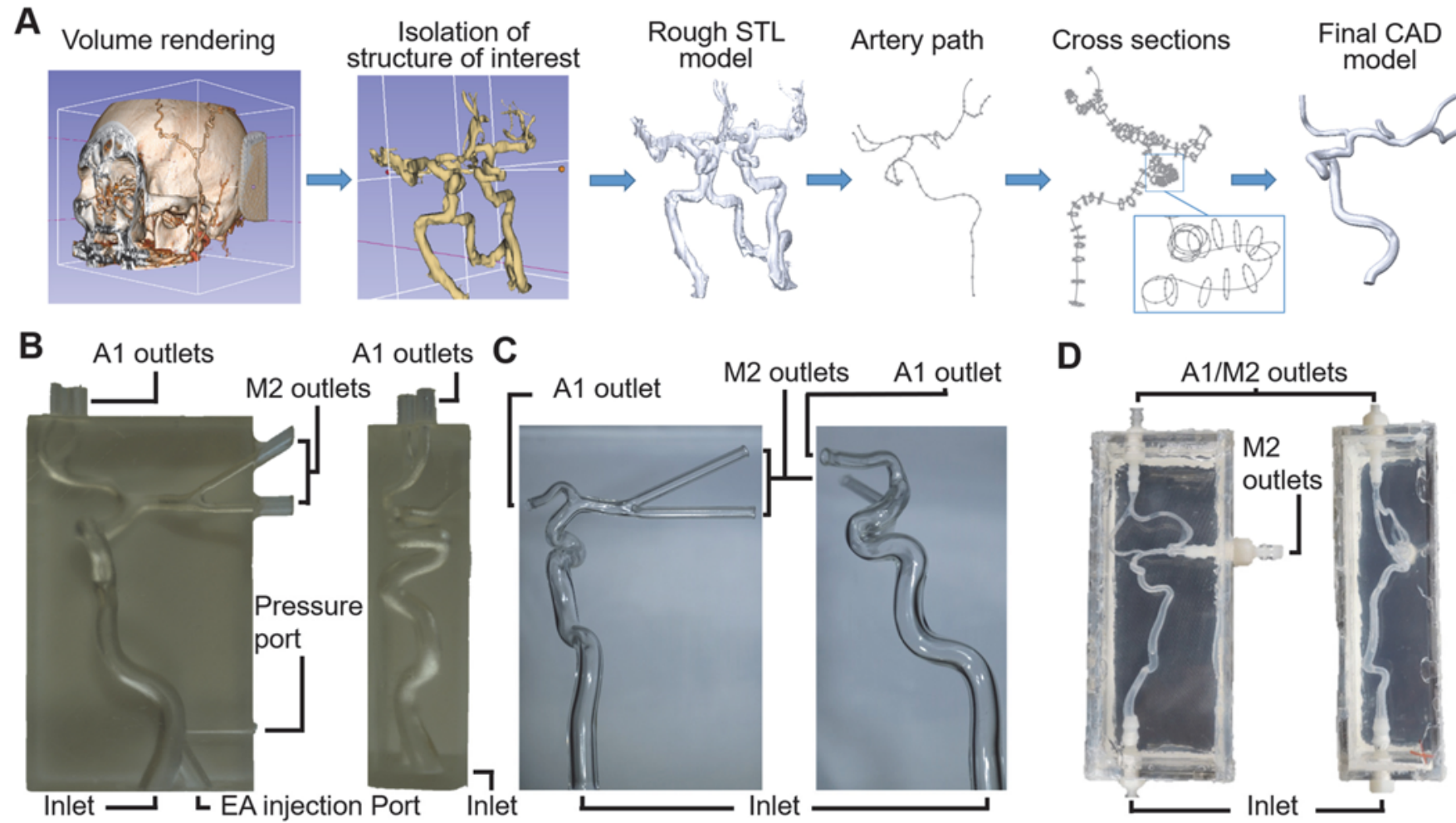

FIG. 1. Development of custom-made cerebrovascular phantoms. A: The unidentified CTA 3D renderings were obtained from patients by using 3D Slicer, and the structure of interest was isolated and converted to STL format. The STL file was then further processed and smoothened in SolidWorks to create the final CAD model. B-D: With this model, 3 transparent phantoms were manufactured: 3D-printed block (B), glass phantom (C), and flexible silicone phantom embedded in Head Gel (D). Inlets and outlets were added to the phantoms for tubing connection, pressure reading, and EA injection. Figure is available in color online only.

sheet. Quarter-inch holes were laser cut for tube-to-male and tube-to-female threaded pipe straight adapters to function as inlet and outlet ports. The silicone phantom was then attached to the tube connection ports on the inside of the acrylic box, which was then lined with clear neutral-cure silicone for stronger sealing. Head Gel (Vascular Simulations), a clear, 2-part mixture that simulates the brain turgor surrounding the arterial phantoms during endovascular procedures, was mixed together with a paint mixer and placed in the refrigerator at $4^{\circ} \mathrm{C}$ for 24 hours to remove the air bubbles. The Head Gel was then poured into the acrylic box and the newly generated air bubbles due to pouring were aspirated using a syringe. The lid of the box was fixed and sealed with neutral-cure silicone lining and stored in a refrigerator at $4^{\circ} \mathrm{C}$ to further remove air bubbles. The cost of a custom-made phantom of the cervical ICA, the ICA terminus, and the MCA candelabra, including $\mathrm{M}_{3}$ branches, is approximately US $\$ 1500$.

\section{Hydraulic System}

The aforementioned cerebrovascular phantoms were connected to a hydraulic system composed of a syringe pump, tubing, and variable flow resistor to replicate intraluminal pulsatile physiological flow rate and pressure (Fig. 2A).

\section{Syringe Pump}

A Legato 270 syringe pump (KD Scientific) was used to deliver physiological flow to the cerebrovascular phantoms. The syringe pump can adjust the stroke volume and flow rate independently to match the heart rate and flow rate simultaneously. For the commonly used rotary pumps, the stroke volume is fixed for a specific size of tube and the flow rate is a linear function of the pulse frequency, making it difficult to match both the heart rate and flow rate at the same time. In this study, the heart rate was 60 beats per minute and the target flow rate was $265 \pm 62$ $\mathrm{mL} / \mathrm{minute}$ for the $\mathrm{ICA}^{5}$ and $121 \pm 28 \mathrm{~mL} /$ minute for the MCA. ${ }^{6}$ Values are expressed as the mean \pm SD.

Using the syringe pump, the 3D-printed and the glass phantoms were pressurized with saline solution. The flexible silicone phantom was pressurized with replicator fluid (Vascular Simulations) per the manufacturer's recommendation. The replicator fluid is slippery and was used to simulate the friction between blood vessels and endovascular devices.

\section{Tubing}

Polyvinyl chloride (PVC) tubing that replicates the vascular anatomy from the femoral endovascular access point to the ICA was fabricated and used to connect the syringe pump to the cerebrovascular phantom (Fig. 2B). The tubing was heat treated and formed to a planar geometry that mimicked the tortuosity and diameters of the human vasculature: femoral and iliac artery $(9.5-\mathrm{mm}$ inner diameter [ID]); aorta (19.1-mm ID); brachiocephalic artery (12.7- 
A

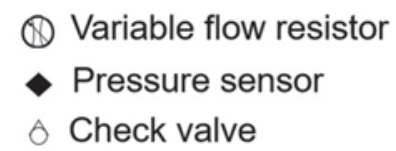

$\Rightarrow$ Fluid flow direction

$\Rightarrow$ Flow to reservoir

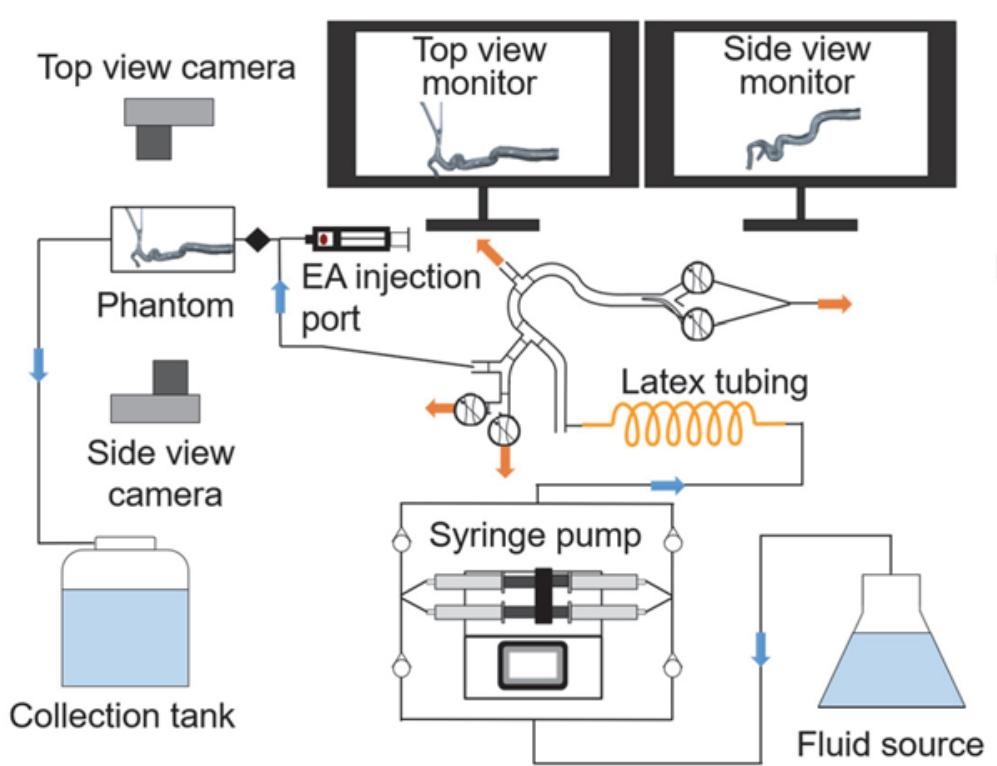

B

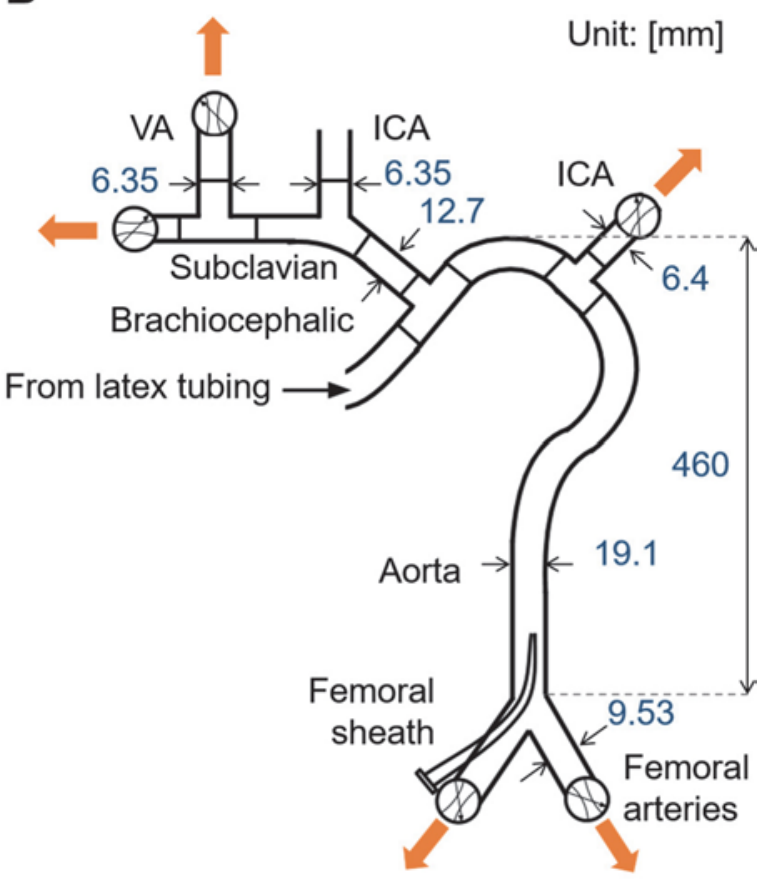

FIG. 2. A: Test bed for mechanical thrombectomy with LVO. The setup mimics the common operation setup of an angiosuite. The operator stands near the femoral arteries and faces 2 monitors displaying the top and side views of the phantom recorded in real time with 2 cameras. The operator accesses the tubing system through the femoral sheath and navigates the device to the phantom found on the left; this simulates the patient with head lying to the left of the access point. The system is pressurized by a syringe pump, which is connected to the ascending aorta through latex tubing. The latex tubing adds compliance to the system, and the length can be modified to adjust the pressure's pulsatility. The fluid cycles through the phantom and into a collection tank. An EA is introduced from the EA injection port. B: The dimensions of the tubing, in millimeters, mimic the dimensions of the cardiovascular system from the groin to the arteries of the neck. Hose clamps are used as variable flow resistors to adjust the flow pressure to replicate physiological flow. VA = vertebral artery. Figure is available in color online only.

mm ID); and carotid artery (6.4-mm ID). In addition, 4 variable-flow resistors and a segment of compliant latex tubing were added to the inlets and outlets of the vascular anatomy, respectively, to adjust the flow pressure and pulsatility. Systolic and diastolic pressures were calibrated by adjusting the variable-flow resistors and the length of flexible soft latex tubing. The latex tubing can also dampen the pressure pulsatility created by the pump. Pressures were calibrated to those recorded clinically with patients in a supine position. Pressure was measured proximal to the ICA by using a differential pressure sensor (model 015PDAA5; Honeywell).

\section{Embolus Analogs}

Two types of EAs were created to study the embolusdevice interaction: the elastic emboli and the fragmentprone emboli. EAs must be capable of causing embolic occlusions and of withstanding the physiological flow in the cerebrovascular phantoms. The EAs were fabricated by inducing coagulation of a mixture of red blood cells (RBCs) and plasma, which were obtained from the University of Michigan Hospital Blood Bank.

EA No. 1 mimics the elastic emboli. Plasma, RBCs, and calcium chloride solution $(2.27 \%$ wt) were mixed at an $8: 2: 1$ volume ratio. ${ }^{-9}$ The mixture was stirred for 2 minutes and transferred to a $15-\mathrm{mL}$ conical centrifuge tube.

EA No. 2 mimics the fragment-prone emboli. Plasma and RBCs were mixed at a 4:1 volume ratio. For every 10 $\mathrm{mL}$ of blood mixture, $1 \mathrm{~g}$ of barium sulfate was added..$^{10}$ This mixture was stirred for 2 minutes. A $1-\mathrm{mL}$ solution composed of $0.9 \mathrm{~mL}$ calcium chloride solution $(2.27 \% \mathrm{wt})$ and $0.1 \mathrm{~mL}$ of $25 \mathrm{NIH}$ Units (NIHU) thrombin was added and briefly vortexed to induce clotting.

For both types of EAs, the tube was stored in water at $37^{\circ} \mathrm{C}$ for 90 minutes. Prior to their introduction into the test bed, the EAs were compressed in a paper towel with a 300 -g mass for 30 seconds.

The tensile properties of the EAs were quantified by tensile tests previously proposed by our group. ${ }^{11}$ These properties have been shown to be the most clinically relevant for understanding the embolus-device interaction. ${ }^{11}$ For each EA type, 4 EAs were tested.

\section{Embolic Occlusion of Cerebral Artery}

The EAs were cut into discs $15 \mathrm{~mm}$ in diameter and $5-8 \mathrm{~mm}$ in thickness. These were introduced into a PVC tube (ID $6.35 \mathrm{~mm}$ ) via syringe negative pressure and slowly injected into the cerebrovascular phantoms through the injection port. After introduction, physiological flow was activated to embolize the EA material downstream. 
A

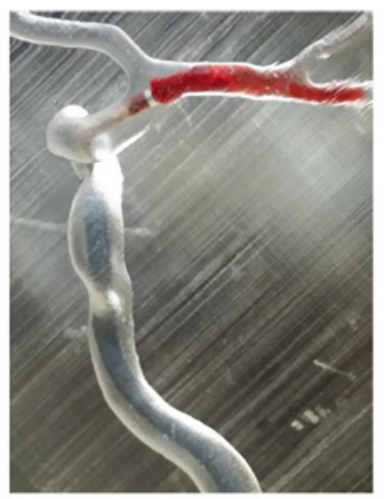

E

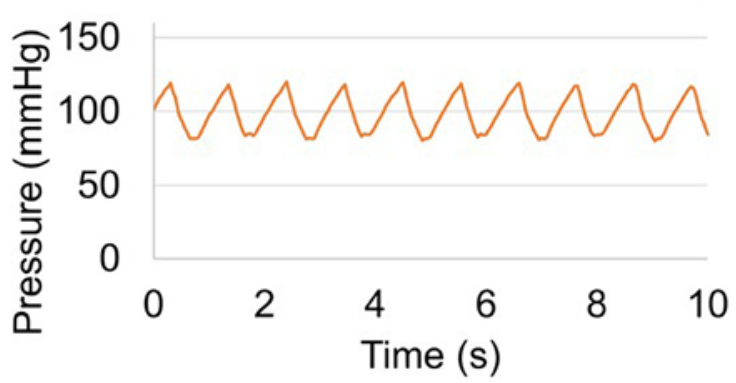

G

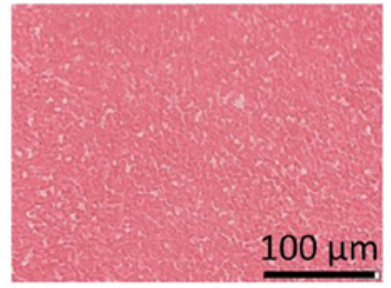

B

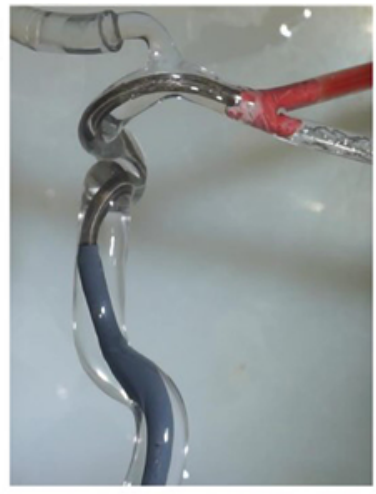

C

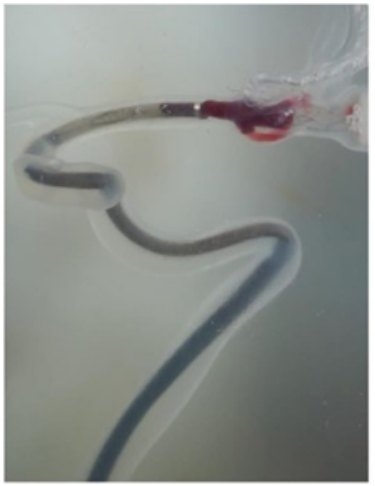

D

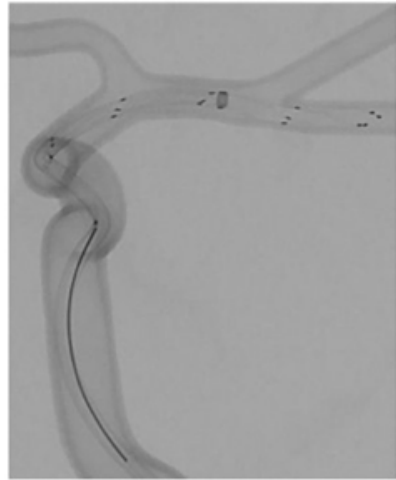

$F$

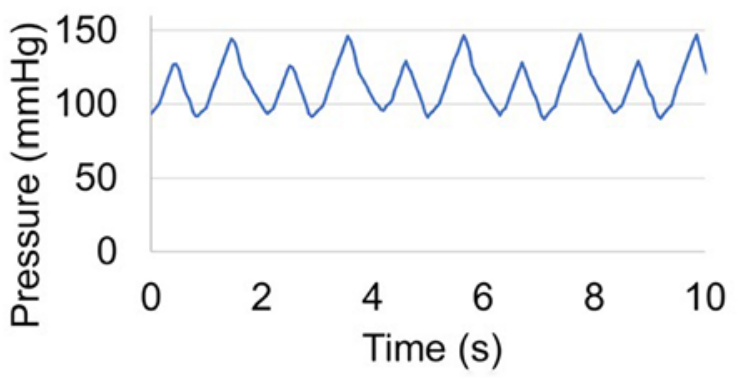

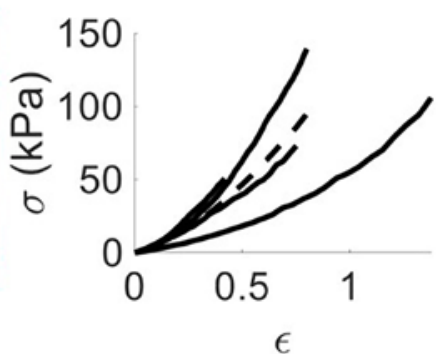

H
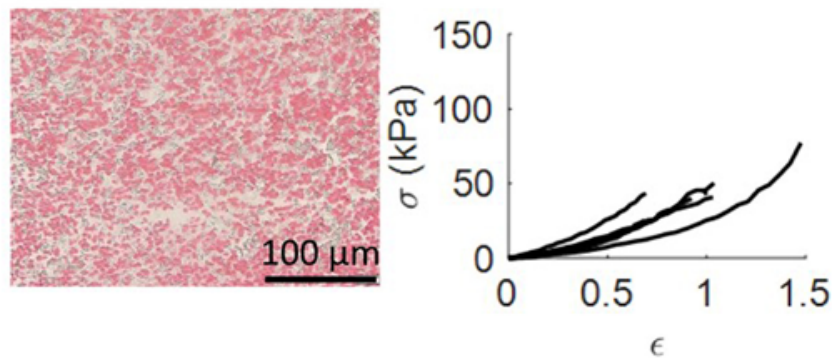

FIG. 3. Removal of a fragment-prone EA by using the CAPTIVE technique inside the 3D-printed block phantom (A), glass phantom (B), and flexible silicone phantom (C). The glass phantom has similar visualization (D) under radiography compared to the clinical setting. The endovascular test bed generated physiological blood pressure of $119 / 79 \mathrm{~mm} \mathrm{Hg}(\mathrm{E})$ to simulate the healthy patient and $150 / 90 \mathrm{~mm} \mathrm{Hg}(\mathbf{F})$ to simulate the stroke patient. Tensile test results are shown for elastic $E A(\mathbf{G})$ and fragment-prone $E A(H)$. The dashed line is the average of the 4 repeated tensile test results (solid lines). Figure is available in color online only.

\section{Biaxial Imaging System}

The optically transparent phantoms enabled conventional cameras to visualize the embolus-device interaction without the need of radiation. Two cameras (model a6000; Sony) were used in top and side projections to record digital video of the mechanical thrombectomy process. Videos were stored and projected in real time on 2 wide-field high-definition displays placed at the femoral access site (Fig. 2A). Angiography of cerebrovascular phantoms and thrombectomy was performed with biplane fluoroscopy (Artis zee, Siemens).

\section{Mechanical Thrombectomy of Embolic Occlusion of MCA}

An 8-Fr femoral sheath (Pinnacle, 10 or $25 \mathrm{~cm}$; Terumo) was introduced into the PVC tubing at the level corresponding to the right femoral artery, and a 5-Fr vertebral catheter was advanced over a guidewire into the aorta. The PVC tube that led to the arterial phantom was then selected over the wire, and the vertebral catheter was advanced into the proximal phantom. The vertebral catheter was exchanged over the wire for a 0.088 -inch Neuron Max (Penumbra), which was parked into the proximal phantom. At this point, mechanical thrombectomy was performed with the device and technique of choice. For the purpose of this paper, mechanical thrombectomy was conducted by continuous aspiration prior to intracranial vascular embolectomy (CAPTIVE) technique with a Solitaire Platinum revascularization device (Medtronic) and aspiration catheter (ACE 68; Penumbra) connected to full vacuum of $-650 \mathrm{~mm} \mathrm{Hg}$ (vacuum pump model 01-12-405; Allied Healthcare) (Fig. 3). Thrombectomy procedures were performed with the elastic EAs and the fragment-prone EAs in each phantom. Video 1 shows thrombectomy with elastic EAs in the 3D-printed, glass, and silicone phantoms, respectively, and thrombectomy with the fragment-prone EA in the glass phantom.

VIDEO 1. Mechanical thrombectomy of elastic EAs in 3D-printed, glass, and silicone models of the MCA, and thrombectomy of a fragment-prone analog in the glass phantom. Copyright Luis $\mathrm{E}$. Savastano. Published with permission. Click here to view. 
TABLE 1. Comparison of 3D-printed, glass, and silicone phantoms used in the test bed for mechanical thrombectomy in LVO stroke

\begin{tabular}{lccc}
\hline \multicolumn{1}{c}{ Attribute } & 3D-Printed Phantom & Glass Phantom & Silicone Phantom \\
\hline Similarity to lumen of large cerebral arteries & Good & Good & Good \\
\hline Ability to replicate physiological flow & Very good & Very good & Very good \\
\hline Visualization of embolus-device interaction & Poor & Excellent & Good \\
\hline Navigation of devices to target & More difficult than patient & Easier than patient & Similar to patient \\
\hline Deformation of phantom due to device-transmitted forces during intervention & Absent & Absent & Minor \\
\hline Arterial wall damage & Absent & Absent & Minimal \\
\hline Lumen collapse under vacuum & Absent & Absent & Absent \\
\hline Perforating arteries & Absent & Possible & Absent \\
\hline Lead time & $\sim 2$ days & $5-6$ wks & 3 wks \\
\hline Cost & $<$ US\$30 & $\sim$ US $\$ 3000$ & $\sim$ US\$1500 \\
\hline
\end{tabular}

* Material cost only, excluding costs of printer and labor.

The performance of each phantom was evaluated by a team of mechanical engineers, and the similarity to clinical experience was subjectively rated by 2 fellowshiptrained endovascular neurosurgeons (A.S.P. and L.E.S.) in $>100$ simulated interventions. Scores used for grading included poor, fair, good, very good, and excellent.

The phantoms have shown relatively good longevity during the evaluation. The 3D-printed block phantoms and glass phantoms can probably accommodate several hundred simulations without macroscopic damage or limits in shelf life. The silicone phantoms, however, are subject to wear and tear and possible damage with aggressive device manipulation. In our experience, gentle use of this phantom allows at least 100 thrombectomy interventions.

\section{Results}

A test bed for embolic occlusion of cerebrovascular arteries and mechanical thrombectomy was developed to recreate intervention in patients in a conventional angiosuite situation. The test bed was highly versatile and allowed realistic performance and radiation-free visualization of mechanical thrombectomy for stroke due to large-vessel occlusion (LVO) with rapid exchange of different geometries and phantom types.

\section{Cerebrovascular Phantoms}

The test bed proved highly versatile and allowed for rapid exchange of different geometries and phantom types. Figure 3A-C shows the 3D-printed phantom, glass phantom, and silicone phantom. The attributes of each phantom are compared in Table 1 on the basis of the following features.

1. Ability to represent the lumen of the large cerebral arteries with accurate 3D geometry and smooth surface. Small branching arteries and perforating arteries are generally not included in phantoms because of the difficulty of maintaining a patent lumen in lower-caliber vessels (generally $<1 \mathrm{~mm}$ in diameter).

2. Ability to replicate pressures and flows within targeted physiological ranges.

3. Visualization of embolus-device interaction, which depends on the transparency of the material, the surface texture/roughness, and the ability to freely rotate the phantom to obtain the best angle for visualization (block is less favorable compared to freestanding geometry).

4. Navigation of the device to the target, compared to the average clinical situation, based on subjective visual and haptic feedback of friction and forces during device advancement to target by 2 endovascular neurosurgeons.

5. Deformation of the phantom due to mechanical manipulation of the device during both advancement and withdrawal.

6. Arterial wall damage by the device, including perforation and dissection.

7. Lumen collapse under full vacuum by large-bore suction catheters during mechanical thrombectomy.

8. Ability to include small arterial branches and perforating arteries in the phantom and appropriately mimic occlusion and recanalization phenomena in these vessels.

Both the fragment-prone EA and the elastic EA embolized under physiological flow and consistently lodged at the MCA birfurcation of the manufactured cerebrovascular phantoms (Fig. 3A-C). Emboli remained wedged in the MCA bifurcation for at least 30 minutes under hydraulic stress, demonstrating tolerance to the physiological flow without fragmentation and downstream migration. On interaction with the thrombectomy device, the EA elongated, thinned, and fractured, sometimes leading to embolization; the results are also reported in a previous study. ${ }^{11}$ The fragment-prone EA demonstrated more frequent embolization compared to the elastic EA.

With fluoroscopy, the silicone and glass phantoms provided good visualization of the arterial geometry, whereas the arterial lumen within the 3D-printed block could not be identified unless the roadmap function (after iodinated contrast injection) was used. The visualization of the thrombectomy devices within the different phantoms was overall similar and equivalent to the clinical setting. Figure 3D shows the fluoroscopic view of the glass phantom with a suction catheter, microcatheter, and stent retriever placed inside. 


\section{Pressure Results}

Accurate physiological pressures with pulsatile waveforms were generated to simulate healthy patients $(120 / 80$ $\mathrm{mm} \mathrm{Hg})$ and patients who have suffered stroke (147/85 $\mathrm{mm} \mathrm{Hg}$ ). The endovascular test bed created pressure waveforms of approximately $120 / 80 \mathrm{~mm} \mathrm{Hg}$ for the healthy case (Fig. 3E) and 150/90 mm Hg for the stroke case (Fig. $3 \mathrm{~F})$. Higher systolic pressures up to $200 \mathrm{~mm} \mathrm{Hg}$ could be achieved by adjusting the variable flow resistors and the length of the latex tubing.

\section{Characterization of EAs}

Figure $3 \mathrm{G}$ and $\mathrm{H}$ show the stress $(\sigma)$ versus strain $(\varepsilon)$ relation for the elastic and fragment-prone EAs. The elastic EAs had ultimate tensile strain $\left(\varepsilon_{u t}\right)$ of $0.43-1.44$ (0.86 $\pm 0.42)$ and ultimate tensile stress $\left(\sigma_{u t}\right)$ of $54-149 \mathrm{kPa}(98$ $\pm 43 \mathrm{kPa}$ ). The fragment-prone EAs had $\varepsilon_{u t}$ of $0.69-1.04$ $(0.89 \pm 0.17)$ and $\sigma_{u t}$ of $31-50 \mathrm{kPa}(41 \pm 8.1 \mathrm{kPa})$. Values are expressed as the mean $\pm \mathrm{SD}$.

\section{Discussion}

The ability to recreate highly accurate cerebral artery phantoms and to replicate arterial flow waveforms in vitro is essential to understand the efficacy and failure modes of mechanical thrombectomy devices. Techniques for the manufacture of different types of phantoms and EAs from fresh animal blood do exist in the literature, but they require highly skilled labor and vast experience to be used. Ready-to-use cerebrovascular simulators and emboli are commercially available but at a very high cost. Several institutes across the US offer in-house testing, but this option is not practical for most research projects due to the prohibitive cost (on the order of $>$ US $\$ 15,000$ per session). In this paper we have described in detail all the components necessary to establish and operate a high-fidelity, versatile, radiation-free, and affordable endovascular simulator for LVO stroke (including EAs) with minimal capital investment.

In order to directly visualize the revascularization procedure without the need of a fluoroscopy unit, in this study we described 3 transparent or semitransparent phantoms. All 3 phantoms evaluated in this investigation demonstrated individual strengths and weaknesses. The 3D-printed model most accurately represents the angioarchitecture and is the most accessible option, because 3D printers are widely available and affordable. However, in our experience, navigation of the devices is slightly more challenging than in the equivalent anatomy in patients, despite the coating of the inner lumen with mineral oil to increase lubricity.

We found the glass model easier to navigate compared to equivalent anatomy in patients, and visually this model most clearly reveals the interaction between emboli and devices. The flexible silicone phantom provides the most accurate haptic representation of the navigation of thrombectomy devices in patients, deforming and moving when forces are applied by the device. However, the silicone phantom wall is still significantly more resistant than the cerebral artery and tolerates without tearing forces and movements that would be deemed unsafe in clinical situations.
In our laboratory, it has been common practice to first evaluate a certain cerebrovascular geometry in 3D-printed blocks, given the easy prototyping and rapid iterations. After satisfactory optimization of the geometry (by adding EA delivery and pressure ports, and further refinement to remove unrealistic geometrical features caused by reconstruction), we purchased customized flexible silicone phantoms to test the delivery of a thrombectomy device, and glass phantoms to clearly visualize EA/device interaction. The use of a radiolucent model, which significantly facilitates testing and improves visualization, also allows complementary radiographic angiography, which is critical in the development and simulated use of thrombectomy devices.

Although valuable, the phantoms described here exclusively represent the main cerebral artery lumen and major branching vessels. In our experience it is challenging to include small branches and perforating arteries with lumens $<1 \mathrm{~mm}$, given current manufacturing techniques. For 3D-printed phantoms, the small channel cannot be printed due to the following drawbacks: 1) limited printer resolution, and 2) the resin (printing material) can be trapped inside those small channels during printing and be difficult to remove, leading to obstruction once the resin is cured. For silicone phantoms, the casting material is also difficult to remove from those small channels. For glass phantoms, perforators of approximately $100 \mu \mathrm{m}$ (personal communication with the manufacturer, September 2019) could be manufactured, but we have not personally evaluated this. Therefore, these small vessels were not tested during simulation of cerebrovascular events and interventions. In addition, the ability of these phantoms to mimic the interaction between devices and arterial wall, as well as embolus and endothelium, is unknown. However, given the artificial nature of the phantoms, it is reasonable to estimate that they provide a limited representation of these "biologically" related features. In vivo animal models and ex vivo fresh human brain may provide an accurate biological and anatomical substrate to complement the described endovascular test bed.

Manufacturing techniques to generate EAs are available in the literature.,12-14 These techniques use animal blood and therefore are not readily available, cause animal discomfort, and/or are expensive. In addition, these EAs made from porcine or bovine whole blood are significantly weaker compared to emboli harvested from LVO strokes: $6 \mathrm{kPa}$ and $30 \mathrm{kPa}$, respectively, compared to $\sigma_{u t}$ ranging from 63 to $2396 \mathrm{kPa} \cdot{ }^{13,14}$ The EAs presented in this study are stronger in tension and more similar in strength to emboli from patients with LVO stroke than those in the literature. ${ }^{11}$ EAs must have sufficiently high ultimate tensile stress to withstand physiological pressures, which may have caused the EAs in the literature to fracture after lodging, preventing further mechanical thrombectomy study. The ability to withstand physiological pressures makes the stronger EAs in this study better for the mechanical thrombectomy test bed than published animal EAs, which are significantly weaker than emboli in patients with LVO. ${ }^{11}$

Although these emboli are valuable and easy to make, there are 2 limitations to the use of the EAs presented in this study as realistic models for emboli in stroke patients. 
First, the EAs described here, like the ones described in the published literature, are mostly histologically homogeneous. Our group has shown that patients' emboli are frequently heterogeneous, which may lead to more embolization during mechanical thrombectomy. To mitigate this limitation, we engineered EAs to mimic the tensile properties of human emboli, and we included a fabrication technique for fragment-prone EAs. Second, the EAs' strengths do not represent the whole spectrum of emboli from patients with LVO stroke. A recent paper analyzing patient emboli harvested during LVO technical thrombectomy revealed a large range of tensile properties, with $\varepsilon_{u t}$ ranging from 1.05 to $4.89(2.41 \pm 1.04)$ and $\sigma_{u t}$ ranging from 63 to $2396 \mathrm{kPa}(569 \pm 695 \mathrm{kPa}){ }^{11}$ whereas $\sigma_{u t}$ in our study was $<150 \mathrm{kPa}$. A range of EAs that can mimic the broad histological and mechanical spectra of emboli encountered in patients with stroke is needed to further advance thrombectomy research. Finally, although this paper is dedicated to describing this platform for the testing of devices for mechanical thrombectomy in stroke, we believe that the platform could also have value in education and training. The platform enables interventions guided by concurrent direct visualization and fluoroscopy, allowing the trainee to better appreciate in real time the interactions of emboli, devices, and phantoms, while using real devices with realistic haptic feedback.

\section{Conclusions}

The test bed presented in this study is a comprehensive, realistic, and versatile platform that enables high-quality analysis of embolus-device interaction in multiple cerebrovascular phantoms and EAs. However, there are critical features determining the efficacy and risk profile of thrombectomy devices that cannot be appropriately tested, including the deformation of phantoms to device-transmitted forces, wall damage, luminal collapse, adhesion of the embolus to the phantom surface, and recanalization of small branches and perforating arteries. Optimized phantoms, or hybrid test beds in which biological tissues are used, should be developed and validated to improve our capacity to conduct research in mechanical thrombectomy in stroke.

\section{Acknowledgments}

Sources of support include the U-M Coulter Translational Research Partnership Program and the Centers for Accelerated Innovations of the National Heart, Lung, and Blood Institute, NIH.

\section{References}

1. Chueh JY, Wakhloo AK, Gounis MJ. Effectiveness of mechanical endovascular thrombectomy in a model system of cerebrovascular occlusion. AJNR Am J Neuroradiol. 2012;33(10):1998-2003.

2. Mokin M, Setlur Nagesh SV, Ionita CN, et al. Comparison of modern stroke thrombectomy approaches using an in vitro cerebrovascular occlusion model. AJNR Am J Neuroradiol. 2015;36(3):547-551.

3. Ionita CN, Mokin M, Varble N, et al. Challenges and limitations of patient-specific vascular phantom fabrication us- ing 3D Polyjet printing, in Molthen RC, Weaver JB (eds): Proceedings of the Society of Photo-Optical Instrumentation Engineers. SPIE Medical Imaging, vol 9038; 2014. doi:10.1117/12.2042266

4. Boularas D, Cheban D. Asymptotic stability of switching systems. Electron J Differ Equ. 2010;2010 (21):1-18. http:// ejde.math.txstate.edu. Accessed February 17, 2020.

5. Schöning M, Walter J, Scheel P. Estimation of cerebral blood flow through color duplex sonography of the carotid and vertebral arteries in healthy adults. Stroke. 1994;25(1):17-22.

6. Weiss A, Beloosesky Y, Kenett RS, Grossman E. Change in systolic blood pressure during stroke, functional status, and long-term mortality in an elderly population. Am J Hypertens. 2016;29(4):432-438.

7. Duffy S, Farrell M, McArdle K, et al. Novel methodology to replicate clot analogs with diverse composition in acute ischemic stroke. J Neurointerv Surg. 2017;9(5):486-491.

8. Gunning GM, McArdle K, Mirza M, et al. Clot friction variation with fibrin content; implications for resistance to thrombectomy. J Neurointerv Surg. 2018;10(1):34-38.

9. Liu Y, Zheng Y, Li AD, et al. Cutting of blood clots-experiment and smooth particle Galerkin modelling. CIRP Ann. 2019;68:97-100.

10. Chueh JY, Puri AS, Wakhloo AK, Gounis MJ. Risk of distal embolization with stent retriever thrombectomy and ADAPT. J Neurointerv Surg. 2016;8(2):197-202.

11. Liu Y, Zheng Y, Reddy AS, et al. Analysis of human emboli and thrombectomy forces in large-vessel occlusion stroke [published online February 28, 2020]. J Neurosurg. doi:10.3171/2019.12.JNS192187

12. Chueh JY, Wakhloo AK, Hendricks GH, et al. Mechanical characterization of thromboemboli in acute ischemic stroke and laboratory embolus analogs. AJNR Am J Neuroradiol. 2011;32(7):1237-1244.

13. Krasokha N, Theisen W, Reese S, et al. Mechanical properties of blood clots - a new test method. Materialwiss Werkstofftech. 2010;41(12):1019-1024.

14. Malone F, McCarthy E, Delassus P, et al. The mechanical characterisation of bovine embolus analogues under various loading conditions. Cardiovasc Eng Technol. 2018;9(3):489502.

\section{Disclosures}

The authors report no conflict of interest concerning the materials or methods used in this study or the findings specified in this paper.

\section{Author Contributions}

Conception and design: Savastano, Reddy, Liu, Cockrum. Acquisition of data: Reddy, Liu, Cockrum, Gebrezgiabhier, Davis. Analysis and interpretation of data: Reddy, Liu. Drafting the article: Savastano, Reddy, Liu, Cockrum. Critically revising the article: Savastano, Reddy, Liu, Zheng, Pandey, Shih. Reviewed submitted version of manuscript: all authors. Approved the final version of the manuscript on behalf of all authors: Savastano. Administrative/technical/material support: Savastano, Pandey, Shih. Study supervision: Savastano, Pandey, Shih.

\section{Supplemental Information \\ Videos}

Video 1. https://vimeo.com/390998020.

\section{Correspondence}

Luis E. Savastano: Mayo Clinic, Rochester, MN. savastano.luis@ mayo.edu. 